\title{
Optimal ATC Enhancement Model: Analysis of the Effect of Thyristor-Controlled Series Compensation
}

\author{
Naresh Kumar Yadav \\ Department of Electrical Engineering, \\ DeenbandhuChhotu Ram University of Science \& Technology, \\ Murthal (Sonepat)-Haryana, India \\ nareshyadhavdr@gmail.com
}

\begin{abstract}
In the functioning of the deregulated system, the ATC is considered as one of the demanding criteria. Generally, the maximum demand for enhancing the ATC is done by exploiting FACTS devices in the power system. Nevertheless, it undergoes severe problem while determining the best position as well as the recompense phase of FACTS. In this paper, TCSC devices are used in order to recompense the restriction of FACTS. In addition, a new Hybrid Grey Wolf Optimization and Flower Pollination Algorithm (HGWFPA) are presented for ATC enhancement. Finally, the simulations are performed on three standard bus systems namely IEEE 30, IEEE 24, and IEEE 57. Moreover, the proposed HGWFPA method is compared with the conventional GWO and FPA method. Ultimately, the experimentation shows that the efficiency of the proposed techniques in terms of ATC enhancement.
\end{abstract}

Keywords: FACTS, ATC, TCSC, power loss, IEEE bus system

\begin{tabular}{ll} 
Nomenclature \\
\hline Abbreviations & Descriptions \\
\hline ATC & Available transfer capability \\
GA & Genetic Algorithm \\
PSO & Particle Swarm Optimization \\
ACO & Ant Colony Optimization \\
GSA & Gravitational Search Algorithm \\
TLBO & Teaching Learning Based Optimization \\
TCSC & Thyristor Controlled Series Capacitor \\
BBO & Biogeography-Based Optimization \\
MDE & Modified Differential Evolution \\
LFC & Load Frequency Control \\
DE & Differential Evolution \\
OPF & Optimal Power Flow \\
APSOA & Adaptive Parallel Seeker Optimization Algorithm \\
CPF & Continuation Power Flow \\
CSO & Cat Swarm Optimization \\
OASIS & Open Access Same Time Information System \\
ETC & Existing Transmission Commitments \\
DE & Differential Evolution \\
PID & Proportional Integral Derivative \\
TTC & Total Transfer Capability \\
CBM & Capacity Benefit Margin \\
TRM & Transfer Reliability Margin \\
SVM & Support Vector Machine \\
GWO & Grey Wolf Optimization \\
SVC & Static VAR Compensator \\
UPFC & Unified Power Flow Controller \\
\hline
\end{tabular}




\section{Introduction}

In the power system, to maintain and assure the power transactions as well as consistent operation of the networks the transmission network owners offer to unbundle services in an open market environment [1]. In order to implement open access [2], ATC is utilized to notify the entire contributor to the energy market of a power system. Because of dynamic and static security constraints, the endeavor of ATC computation of a non-linear power system creates two main disadvantages such as calculating accuracy and speed [3].

Nowadays, the approaches for calculating the transfer capability are developing. However, the approaches exploited currently are oversimplified, which do not regard as the effects namely system policies, nonlinearities, interactions among the power transfers and loop flows [13] [14]. Under an open market environment, an effectual and efficient calculating tool used for precise computation of ATC that is greatly necessary for all transmission providers. Although an important development is done in developing such tools, the main confront enduring is to decide ATC precisely in unreliable load circumstances that consider static and dynamic security limits [26].

Generally, FACTS devices provide an efficient as well as a promising substitute for existing approaches for ATC enhancement. Moreover, it offers new control services, both in dynamic stability control steadystate and power flow control [10] [11] [12]. In the electric power system, controlling power flow without generation rescheduling or topological modifications is able to enhance the network performance significantly [16] [17]. Through various case studies, the result of an SVC and TCSC on the ATC enhancement are examined and verified with the appropriate location. It is revealed while installing SVC in the proper position will enhance voltage profile, ATC, and TCSC.

While comparing with other FACTS devices, the TCSC is considered as one of the fast response devices with lower costs [19]. Also, TCSC is considered as one of the most important FACTS devices that can installed to enhance the transient stability, enhance the system loadability minimize transmission loss, and maximize the power transmission capacity. At the well-tuned parameters, the TCSC required to be optimally installed within the suitable network in order to attain the previous advantages [18]. Subsequent objectives are considered for optimal installation of TCSC devices: reducing reactive as well as active power losses, enhancing power transmission capacity and stability margin [23] [24] [25].

In recent years, optimization approaches are well developed and attracts many researchers. It is because of their huge contribution and impacts in the field of economics and designing [20]. However, highly developed optimization tools are frequently required due to the increased complexity of real-world issues [21] [22]. In order to solve the complicated mathematical models, the meta-heuristic optimization techniques turn into a widespread option. Metaheuristic optimization approaches comprise numerous techniques in order to attain the optimal solution such as GA [31], PSO [32], BBO, ACO, and GSA [33].

The main aim of this paper is to present an HCSGWO approach to resolve the optimization issues in the power system. In addition, the presented technique is used to optimize the utmost ATC in the power transmission system. Furthermore, TCSC devices are exploited in this paper in the place of FACTS devices.

\section{Literature Review}

In 2017, Rahul Agarwal et al [1], proposed a novel optimization method known as TLBO to find out the optional rating and location of the TCSC for the power system. Moreover, this paper exhibits a generalized technique for the optimization TLBO algorithm, by considering the end objective, an enhanced voltage profile, and power transfer capacity was attained. Finally, from the result, it was clear the minimization of active and reactive power loss of the line was attained after the positioning of TCSC in the power system.

In 2018, Dillip Kumar Sahoo et al [2] presented an MDE method for LFC of interconnected power system by considering the nonlinearity. Also, by exploiting various schemes of the DE approach the increases of the fuzzy PID controller were optimized. Subsequently, changing in DE method was presented for the optimal scheme using an easy through the effectual method of modifying two of its significant control parameters such as crossover probability and step size with an intention of attaining enhanced performance. In addition, a TCSC algorithm was introduced that was appropriate for the LFC issue. Finally, the performance of the fuzzy PID controller synchronized with TCSC was examined under arbitrarily load.

In 2019, M.B. Shafik et al [3] presented the framework named as OPF to locate the optimal site as well as the size of the TCSCs devices. For the minimization of generation cost and installed TCSC device cost the techno-economic problems were considered. Additionally, an APSOA was examined to use the study of techno-economic. The multi-objective OPF issue was solved by exploiting the presented APSOA, whereas the search space was reduced by LSR. At normal as well as contingency operating circumstances, the proposed method was examined against three IEEE standards with 9, 30 and 57-bus test systems. To 
reveal the capability and increases of the proposed algorithm, four-study cases were considered to reduce losses and total voltage deviation.

In 2017, O. Ziaee no et al [4] developed a novel method for optimal position allocation for TCSC in power transmission network. Here, mainly two-stage stochastic procedures were considered. In the first phase, the optimal positioning, as well as an upper limit was identified based on the number of TCSCs. The AC viability of the solution that was attained in the first phase for different load cases was checked in the second phase regarding load uncertainties. To solve the issue iteratively, a comprehensive Benders decomposition method was utilized, which consists of both the active and reactive power flow of the transmission network. To examine the reliability of the proposed process and to improve the perceptive of the TCSC position-allocation issue the IEEE 118-bus was exploited.

In 2017, T. Nireekshana et al [5] worked on improvement and determination of ATC, which was a significant problem in the deregulated operation of the power systems. Here, the employ of FACTS devices namely TCSC and SVC was examined in normal and contingency circumstances so as to improve power transfer transactions. However, ATC was calculated by means of CPF approach taking into consideration of both the voltage profile and thermal limits. In order to resolve the controlling and location parameters of TCSC and SVC, the CSO was exploited as an optimization tool. For standard and different contingency scenarios, the recommended method was examined on IEEE 14 as well as IEEE 24-bus reliability test system.

In 2017 M. Venkateswara Rao et al [6] worked on a method, which was to estimate the ATC by exploiting sensitivity factors technique. Furthermore, the ATC value was improved by exploiting OPF and FACTS controllers namely STATCOM, UPFC, and SSSC. Hence, a novel current method on the basis of modeling was developed for the represented FACTS controllers. The computational load, as well as the time, was taken for convergence, which was minimized by means of proposed modeling. While compared with the normal load flow, the system ATC was improved by exploiting OPF, which was identified from the analysis.

In 2017, Ashwani Kumar and Jitendra Kumar [7] developed a method for the ATC improvement by means of optimal power flow. To find out the enhancement of ATC, the Z states Constant Impedance load, I states for Constant Current load, and P states Constant Power load (ZIP) load method with the constant power load was integrated into an OPF method that identifies the impact of ZIP load method. In order to identify the impact of the ATC with and without FACTS devices, the various amalgamations of coefficients of load were represented. On the basis of the outcomes attained, it was finalized that the ATC increases in the occurrence of FACTS devices for all types of transactions under the whole as well as line contingency scenarios.

In 2018, Zora Luburic and Hrvoje Pandzic [8] worked on four different unit commitment methods, which consider energy storage as well as FACTS devices. By using both the technologies, the wind limitation was efficiently minimized and the storage of energy was high effectual at minimizing system operating costs than the FACTS devices. On the other hand, the efficiency of energy storage depends on the wind profile at minimizing system operating costs and wind limitation drastically. Although an enormous amount of these devices was required to efficiently control power flows in the entire system. Line loadings maximization can be extensively performed by FACTS devices.

\section{Basic Description of ATC Enhancement}

\subsection{Objective Function}

Consider a standard bus system, which connected with some external devices. The ATC needs to estimate for the power system. Moreover, this paper mainly focused on the ATC estimation of the system, which available with TCSC. As the number of TCSC to be connected depends on the users, so the need for external devices under such condition is less. Eq. (1) indicates the objective problem model, whereas $\mathrm{L}_{\mathrm{t}}$

represents the group of line indices and $\mathrm{C}_{\mathrm{t}}$ represents the compensation level of $\mathrm{t}^{\text {th }}$ TCSC in the bus system, $\left[\mathrm{L}_{\mathrm{t}}^{*}, \mathrm{C}_{\mathrm{t}}^{*}\right]$ indicates the optimal link in the group of line.

$$
\left[\mathrm{L}_{\mathrm{t}}^{*}, \mathrm{C}_{\mathrm{t}}^{*}\right]=\underset{\left[\mathrm{L}_{\mathrm{t}}, \mathrm{C}_{\mathrm{t}}\right]}{\arg \max } \mathrm{ATC} ; 0 \leq \mathrm{t} \leq \mathrm{N}_{\mathrm{T}}-1
$$

\subsection{Background Illustration}

Nowadays, consumers and producers of the power transmissions are moving in a deregulated way due to the increased demand in electricity. Hence, a single transmission system of power is required to convey power transmissions among the consumer and generator part. Moreover, the quantity of generators is 
varying in electricity markets, which tends to the happening of jamming and overcapacity. In view of the fact that the overloading and congestion are maximized, so the obliteration in the flow of transmission, stability and voltage limits is maximized. However, these problems mainly influence the ATC, because ATC significantly assists to offer security as well as reliability to the power system.

OASIS is kept to update the ATC incessantly, so evaluation of ATC turns into the wearisome part. Generally, "ATC is represented as a measure of the transfer capacity that remains in the physical transmission network for additional profitable action above and over previously committed utilizes." Eq. (2) represents the general formulation of ATC, where $\mathrm{V}^{\mathrm{EC}}$ indicates the ETC, $\mathrm{V}^{\mathrm{TC}}$ indicates the TTC, $V^{C M}$ indicates the CBM, and $\mathrm{V}^{\mathrm{TRM}}$ indicates the TRM. The definition of ATC with a clear description of TTC, TRM, ETC, and CBM is shown in fig. 1.

$$
\mathrm{V}^{\mathrm{ATC}}=\mathrm{V}^{\mathrm{TC}}-\mathrm{V}^{\mathrm{TRM}}-\left(\mathrm{V}^{\mathrm{EC}}+\mathrm{V}^{\mathrm{CM}}\right)
$$

TRM: TRM determines the transmission capacity of the power transfer that is necessary to set up the protection of the interrelated system. The power system can be connected with a small number of uncertainties, which continue in the system for the above measurement.

CBM: CBM determines the transmission capacity of power transfer by means of fulfilling the requirements of consistent generation. Moreover, the components accessible for lessening the load are extremely exploited to function in the interconnected system.

TTC: TTC determines the total power, which conveyed by the system, hence that the whole set of distinct pre- and post-contingency system conditions are fulfilled.

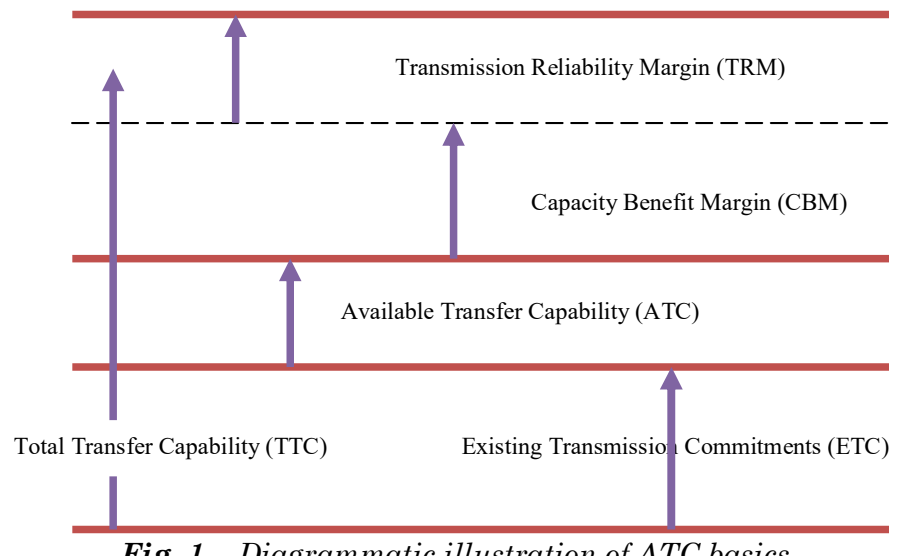

Fig. 1. Diagrammatic illustration of ATC basics

In the past few years, there have been numerous experiments connected with the augmentation of ATC that is reviewed by various researchers. For ATC determination, the curve of the protection border was represented in [24]. Moreover, the ATC using the SVM was estimated in [25]. In view of the fact that the introduction of methods for the estimation of ATC is maximized day by day however their illusory methods are classified into two kinds: on the basis of distribution factors and CPF [26]. The aforesaid techniques are exploited on the condition of AC or DC power flows. On the other hand, this simulation is not successful because it does not require any breakdown and difficulty to create the precise outcome for the general operation of ATC determination [14].

Additionally, the CPF techniques are mostly implemented while they exploit the augmentation of linear deviation by the class of buses to attain the best value [29]. Consequently, under CPF the controlling parameters connected with the testing are moving in a linear way. Therefore, it needs the modification procedure subsequent to the achievement of each iteration. This paper fully depends on the power flow for the estimation of ATC.

\section{Proposed Optimization Algorithms Adopted for ATC Enhancement}

\subsection{Conventional GWO algorithm}

GWO algorithm is a well known population-based nature-inspired method [27]. Generally, GWO algorithm imitates the grey wolves in the form of utilizing its behavior such as social leadership and hunting method. In order to simulate the command hierarchy, four kinds of grey wolves are exploited. Here, the first three optimal position wolves are represented as $\delta, \chi, \lambda$ and these wolves guides the other wolves $\eta$ of the groups on the way to promising areas of the search space. 
The following mathematical equation is used to compute the encircling behavior of each agent of the grey wolves. Eq. (5) and (6) indicates the vectors pand $\mathrm{n}$.

$$
\begin{aligned}
& \overrightarrow{\mathrm{q}}=\left|\mathrm{n} \cdot \overrightarrow{\mathrm{y}}_{\mathrm{p}}^{\mathrm{t}}-\overrightarrow{\mathrm{y}}^{\mathrm{t}}\right| \\
& \overrightarrow{\mathrm{y}}^{\mathrm{t}+1}=\overrightarrow{\mathrm{y}}_{\mathrm{p}}^{\mathrm{t}}-\overrightarrow{\mathrm{p}} \cdot \overrightarrow{\mathrm{q}} \\
& \overrightarrow{\mathrm{p}}=2 \mathrm{i} \cdot \mathrm{r}_{1} \\
& \overrightarrow{\mathrm{n}}=2 \cdot \mathrm{r}_{2}
\end{aligned}
$$

To scientifically simulate the hunting behavior, $\delta, \chi, \lambda$ have superior knowledge regarding the possible position of prey.

$$
\begin{aligned}
& \overrightarrow{\mathrm{q}}_{\delta}=\left|\overrightarrow{\mathrm{n}}_{1} \cdot \overrightarrow{\mathrm{y}}_{\delta}-\overrightarrow{\mathrm{y}}\right| \\
& \overrightarrow{\mathrm{q}}_{\chi}=\left|\overrightarrow{\mathrm{n}}_{2} \cdot \overrightarrow{\mathrm{y}}_{\chi}-\overrightarrow{\mathrm{y}}\right| \\
& \overrightarrow{\mathrm{q}}_{\lambda}=\left|\overrightarrow{\mathrm{n}}_{3} \cdot \overrightarrow{\mathrm{y}}_{\lambda}-\overrightarrow{\mathrm{y}}\right| \\
& \overrightarrow{\mathrm{y}}_{1}=\overrightarrow{\mathrm{y}}_{\delta}-\overrightarrow{\mathrm{p}}_{1} \cdot\left(\overrightarrow{\mathrm{q}}_{\delta}\right) \\
& \overrightarrow{\mathrm{y}}_{2}=\overrightarrow{\mathrm{y}}_{\chi}-\overrightarrow{\mathrm{p}}_{2} \cdot\left(\overrightarrow{\mathrm{q}}_{\chi}\right) \\
& \overrightarrow{\mathrm{y}}_{3}=\overrightarrow{\mathrm{y}}_{\lambda}-\overrightarrow{\mathrm{p}}_{1} \cdot\left(\overrightarrow{\mathrm{q}}_{\lambda}\right) \\
& \overrightarrow{\mathrm{y}}^{\mathrm{t}+1}=\frac{\overrightarrow{\mathrm{y}}_{1}+\overrightarrow{\mathrm{y}}_{2}+\overrightarrow{\mathrm{y}}_{3}}{3}
\end{aligned}
$$

Here, $\overrightarrow{\mathrm{p}}$ is considered as a random value within the range $[-2 p, 2 p]$. Moreover, the wolves are enforced to attacked the prey during the random value $|\vec{p}|<1$. Here, the exploration ability is considered during searching for prey and the exploitation ability is considered during attacking the prey. The random values $\vec{p}$ are used to enforce the search to diverge from the prey. During $1>|\vec{p}|$, the members of the population are forced to move away from the prey.

\subsection{Conventional FPA algorithm}

In [28], biological flower pollination motivates a novel population-based approach known as FPA. Here, the worldwide pollination represented as cross-pollination and the pollinators follows Levy flights whereas; self-pollination is referred to as the local pollination. The flower constancy is also representing as reproduction probability and it is proportional to the resemblance of the two flowers inapprehensive. This approach uses a switching probability $q \in[0,1]$ to manage among the global and local pollination and also FPA is represented as local and global pollination.

$$
\overrightarrow{\mathrm{y}}_{\mathrm{mn}}^{\mathrm{t}+1}=\overrightarrow{\mathrm{y}}_{\mathrm{mn}}^{\mathrm{t}}+\mathrm{v} \times\left(\overrightarrow{\mathrm{y}}_{\mathrm{ml}}^{\mathrm{t}}-\overrightarrow{\mathrm{y}}_{\mathrm{mp}}^{\mathrm{t}}\right)
$$

Here, $\overrightarrow{\mathrm{y}}_{\mathrm{ml}}^{\mathrm{t}}$ and $\overrightarrow{\mathrm{y}}_{\mathrm{mp}}^{\mathrm{t}}$ are considered as the pollen of various flowers however they are in the similar plant species, whereas $v$ is created from the uniform distribution [0,1]. If $\vec{y}_{m l}^{t}$ and $\vec{y}_{m p}^{t}$ are originated from the similar species an arbitrary walk for a local procedure needs to perform. In the global pollination, the pollens of the flowers are enthused through pollinator's for instance pollens and insects can be passed for extensive distances. Therefore, this procedure assures the reproduction and pollination of the fittest solution and it is denoted as follows.

$$
\overrightarrow{\mathrm{y}}_{\mathrm{mn}}^{\mathrm{t}+1}=\overrightarrow{\mathrm{y}}_{\mathrm{mn}}^{\mathrm{t}}+\alpha \times \mathrm{L}(\gamma) \times\left(\overrightarrow{\mathrm{y}}_{\mathrm{mn}}^{\mathrm{t}}-\mathrm{h}^{*}\right)
$$

At iteration $\mathrm{t}, \mathrm{y}_{\mathrm{j}}$ represents the solution vector in order to control the step size, and $\gamma$ is represented as a scaling factor. To imitate the feature transporting of insects on a long-distance with a variety of length steps the Levy flight is exploited, therefore, $L>0$.

$$
\mathrm{L}=\frac{\gamma \Gamma(\gamma) \times \sin \left(\frac{\pi \gamma}{2}\right)}{\pi \times \mathrm{s}^{\mathrm{j}+\beta}},\left(\mathrm{s}>>\mathrm{s}_{0}\right)
$$

In eq. (16) $\gamma$ represents the standard gamma function, as well as it is applicable for large steps. A variable $p$ represents the switching probability, which is exploited to modify the global pollination to exhaustive local pollination and move backward. 


\subsection{Proposed HGWFPA algorithm}

The proposed method is the hybridization of both the conventional GWO and FPA method and it is named as HGWFPA. It is attained by integrating the functionality of both algorithms. In producing the final best solution to the ATC enhancement, two different approaches comprising exploration and exploitation are involved.

The performance of exploitation in GWO is extended with the achievement of the exploration in FPA to create strength of both algorithms. On the other hand, FPA uses the exploration stage to update the positions. As a result of this modification, the enhancement of ATC is satisfied by using the proposed HGWFPA.

$$
\overrightarrow{\mathrm{q}}_{\delta}=\left\{\begin{array}{l}
\mathrm{v} \times\left(\left|\overrightarrow{\mathrm{n}}_{1} \times \overrightarrow{\mathrm{y}}_{\delta}-\overrightarrow{\mathrm{y}}\right|\right), \quad \operatorname{rand}()<0.5 \\
\alpha \times \mathrm{L}(\beta) \times\left(\left|\overrightarrow{\mathrm{n}}_{1} \times \overrightarrow{\mathrm{y}}_{\delta}-\overrightarrow{\mathrm{y}}\right|\right), \operatorname{rand}() \geq 0.5
\end{array}\right.
$$

In Eq. (17), $\vec{q}_{\delta}$ represents a modified supremacy coefficient from eq. (3) and it is used for the diversity search agent in the proposed method. Similar operation can be performed in $\delta, \chi, \lambda$ which is in eq. (7), (8) and (9). The eq. (18) represents the new agent position.

$$
\overrightarrow{\mathrm{y}}_{1}=\overrightarrow{\mathrm{y}}_{\delta}-\overrightarrow{\mathrm{p}}_{1} \times\left(\overrightarrow{\mathrm{q}}_{\delta}\right)
$$

Where, $\vec{y}_{1}$ is performed for first agent position and the same procedure is performed for second and third agent position $\vec{y}_{2}$ and $\vec{y}_{3}$ from eq. (7) (8) and (9). The following pseudo-code represents the proposed

\begin{tabular}{|c|c|c|}
\hline \multicolumn{3}{|c|}{ Algorithm: Pseudo-code of proposed HGWFPA } \\
\hline \multicolumn{3}{|c|}{ Initialize the parameters } \\
\hline \multicolumn{3}{|c|}{ Population $Y_{j}(j=1,2, \ldots . . n), q, p$ and $c$} \\
\hline \multicolumn{3}{|c|}{ Calculate the fitness of each search agent $\mathrm{f}\left(\mathrm{Y}_{\delta}, \mathrm{Y}_{\chi}, \mathrm{Y}_{\lambda}\right)$} \\
\hline \multicolumn{3}{|c|}{ While $\mathrm{t}<\max$ iteration } \\
\hline & For ever & ch agent \\
\hline & & Obtain the current position by eq. (4) and (13) \\
\hline & End for & \\
\hline & & Using eq. (3), (6) and (17) obtain $\mathrm{n}, \mathrm{p}$ and $\mathrm{q}$ \\
\hline & & Compute the fitness of all search agent \\
\hline & & Update $\mathrm{Y}_{\delta}, \mathrm{Y}_{\chi}, \mathrm{Y}_{\lambda}$ by eq. (18) \\
\hline & & $\mathrm{t}=\mathrm{t}+1$ \\
\hline \multicolumn{3}{|l|}{ End while } \\
\hline Return $\mathrm{Y}_{\delta}$ & & \\
\hline
\end{tabular}
HGWFPA.

\section{Result and Discussion}

\subsection{Simulation Procedure}

The simulation of the ATC enhancement in power system was performed on IEEE 24, 30 and 57 test bus systems. Here, the experimentation was done by exploiting the proposed HGWFPA method. The outcome attained from the proposed HGWFPA was compared with that from the existing GWO [30] and FPA [28]. Generally, as per literature, the enhancement of ATC was validated in two TCSC connections. Though, this paper focused to estimate under-five TCSC connections. Moreover, the statistical analysis of the three bus system was analyzed that are demonstrated in the following sub-sections.

\subsection{Performance Analysis}

In Table 1, the performance analysis of the ATC enhancement in IEEE 24 test bus system by HGWFPA is demonstrated. Here, it shows the information with respect to the line number, the link from the bus to the bus and the compensation. Also, the simulation is performed for a power system with and without TCSC. As a result, in the first connection, the line number of the two TCSCs recommends 26 for GWO, 27 FOR FPA and 23 for HGWFPA, while the compensation level is 0.22 for GWO and -1.26 for FPA and 2.33 for HGWFPA. Hence, the overall analysis illustrates that the proposed technique is better than the existing GWO and FPA technique. 


\begin{tabular}{|c|c|c|c|c|c|c|c|c|c|c|c|c|c|}
\hline \multirow{2}{*}{ TCSC } & \multirow{2}{*}{ Connections } & \multicolumn{3}{|c|}{ 2TCSCs } & \multicolumn{3}{|c|}{$3 \mathrm{TCSCs}$} & \multicolumn{3}{|c|}{$4 \mathrm{TCSCs}$} & \multicolumn{3}{|c|}{$5 \mathrm{TCSCs}$} \\
\hline & & GWO & FPA & HGWFPA & GWO & FPA & HGWFPA & GWO & FPA & HGWFPA & GWO & FPA & HGWFPA \\
\hline & Line no & 26 & 27 & 23 & 22 & 24 & 21 & 21 & 20 & 15 & 15 & & 12 \\
\hline & From bus/ to bus & $13 / 22$ & $12 / 22$ & $6 / 7$ & $22 / 23$ & $21 / 23$ & $11 / 13$ & $12 / 13$ & $11 / 14$ & $5 / 6$ & $22 / 21$ & $22 / 21$ & $12 / 13$ \\
\hline & Compensation (p.u) $\times 10^{-3}$ & 0.22 & -1.22 & -2.33 & -1.33 & 1.46 & 2.03 & 1.32 & 1.233 & 1.254 & 1.345 & 1.678 & 2.890 \\
\hline & Line no & 36 & 33 & 31 & 32 & 31 & 27 & 23 & 22 & 20 & 14 & 13 & 11 \\
\hline & From bus/ to bus & $12 / 14$ & $11 / 12$ & $5 / 9$ & $12 / 15$ & $12 / 15$ & $2 / 5$ & $21 / 23$ & $22 / 25$ & $13 / 15$ & $13 / 15$ & $14 / 16$ & $11 / 12$ \\
\hline & Compensation (p.u) $\times 10^{-3}$ & 2.980 & 2.309 & 2.765 & 1.456 & 1.234 & 2.456 & 1.234 & 1.234 & 2.564 & 1.23 & 1.21 & 2.213 \\
\hline \multirow[b]{2}{*}{3} & Line no & 0 & 0 & 0 & 26 & 23 & 22 & 21 & 20 & 19 & & 12 & 11 \\
\hline & From bus/ to bus & $0 / 0$ & $0 / 0$ & $0 / 0$ & $14 / 17$ & $13 / 16$ & $12 / 13$ & $13 / 18$ & $17 / 19$ & $12 / 15$ & $18 / 21$ & $19 / 22$ & $12 / 13$ \\
\hline \multirow{4}{*}{4} & Compensation (p.u) $\times 10^{-3}$ & 0 & 0 & 0 & 1.24 & 2.23 & 1.26 & -2.24 & -2.13 & -3.21 & -1.21 & 1.23 & 2.234 \\
\hline & Line no & 0 & 0 & 0 & 0 & 0 & 0 & 5 & 4 & 2 & 32 & 33 & 32 \\
\hline & From bus/ to bus & $0 / 0$ & $0 / 0$ & $0 / 0$ & $0 / 0$ & $0 / 0$ & $0 / 0$ & $12 / 15$ & $11 / 16$ & $9 / 10$ & $8 / 10$ & $17 / 18$ & $5 / 9$ \\
\hline & Compensation (p.u) $\times 10^{-3}$ & 0.1 & 0.1 & 0.1 & 0.1 & 0.1 & 0.1 & 2.32 & 2.321 & 2.345 & -0.43 & -0.23 & 0.32 \\
\hline & Line no & 0 & 0 & 0 & 0 & 0 & 0 & 0 & 0 & 0 & 33 & 33 & 32 \\
\hline & From bus/ to bus & $0 / 0$ & $0 / 0$ & $0 / 0$ & $0 / 0$ & $0 / 0$ & $0 / 0$ & $0 / 0$ & $0 / 0$ & $0 / 0$ & $19 / 20$ & $21 / 22$ & $24 / 26$ \\
\hline & Compensation (p.u) $\times 10^{-3}$ & 0.2 & 0.2 & 0.2 & 0.2 & 0.2 & 0.2 & 0.2 & 0.2 & 0.2 & -0.2 & -0.23 & 2.21 \\
\hline \multirow{2}{*}{\multicolumn{2}{|c|}{$\begin{array}{l}\text { ATC (MW) without TCSC } \\
\text { ATC (MW) with TCSC }\end{array}$}} & 21.2 & 21.2 & 21.3 & 21.3 & 21.3 & 21.3 & 21.3 & 21.2 & 21.3 & 21.2 & 21.3 & 21.3 \\
\hline & & 107.4 & 118 & 223 & 221 & 224 & 324 & 213 & 321 & 423 & 521 & 213 & 621 \\
\hline
\end{tabular}

Table 2 shows the performance with respect to the enhancement of ATC of the proposed HGWFPA. The simulation is performed for basic load system without TCSC and the link of the power system with TCSC. Furthermore, under five connections such as 3TCSC, 2TCSC, 4TCSC, and 5TCSC it is carried out. The line number of 2TCSC is 22 for GWO and 11 for FPA. Furthermore, the line number of 3 TCSC is 22 for GWO, 23 for FPA and 13 for under five connections, 4 TCSC is 21 for GWO, 12 for FPA and 12 for HGWFPA, and 5 TCSC connection is 21 for GWO, 22 for FPA and 21 for HGWFPA. The overall analysis shows that the proposed technique performs superior to conventional algorithms.

Table 2. Performance Analysis of ATC enhancement in IEEE 30 test bus system

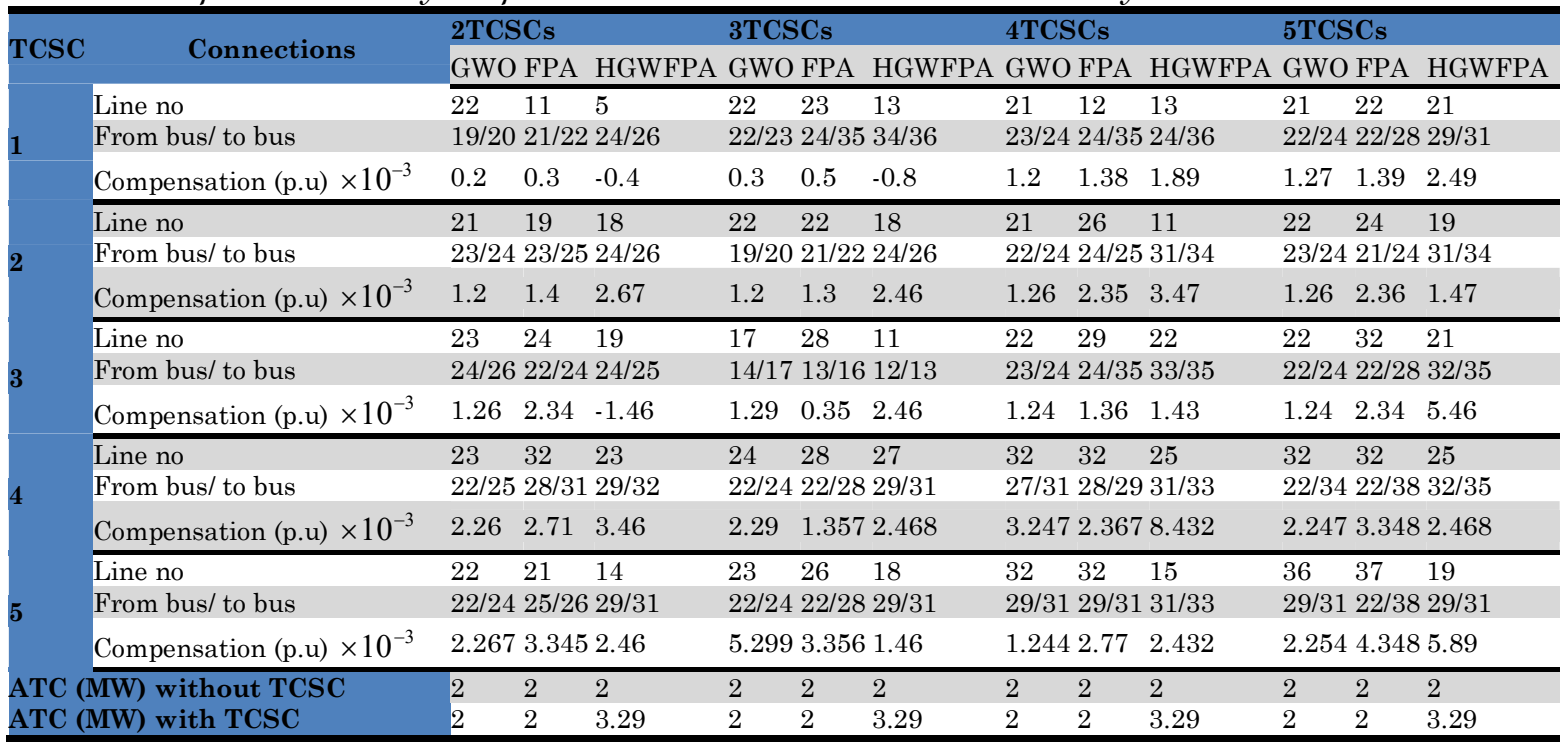

In Table 3, the performance of ATC enhancement for IEEE 57 test bus system is demonstrated. First, the line number for every TCSC link of existing GWO and FPA and proposed HGWFPA is determined. The line number for 3TCSC, 2TCSC, 4TCSC and 5TCSC connections the ATC enhancement without TCSC connection is same for both the conventional and proposed method. However, the enhancement of ATC with TCSC connection for 3TCSC, 2TCSC, 4TCSC, and 5TCSC of proposed HGWFPA is far superior to existing GWO and FPA. 
Table 3. Performance Analysis of ATC enhancement in IEEE 57 test bus system

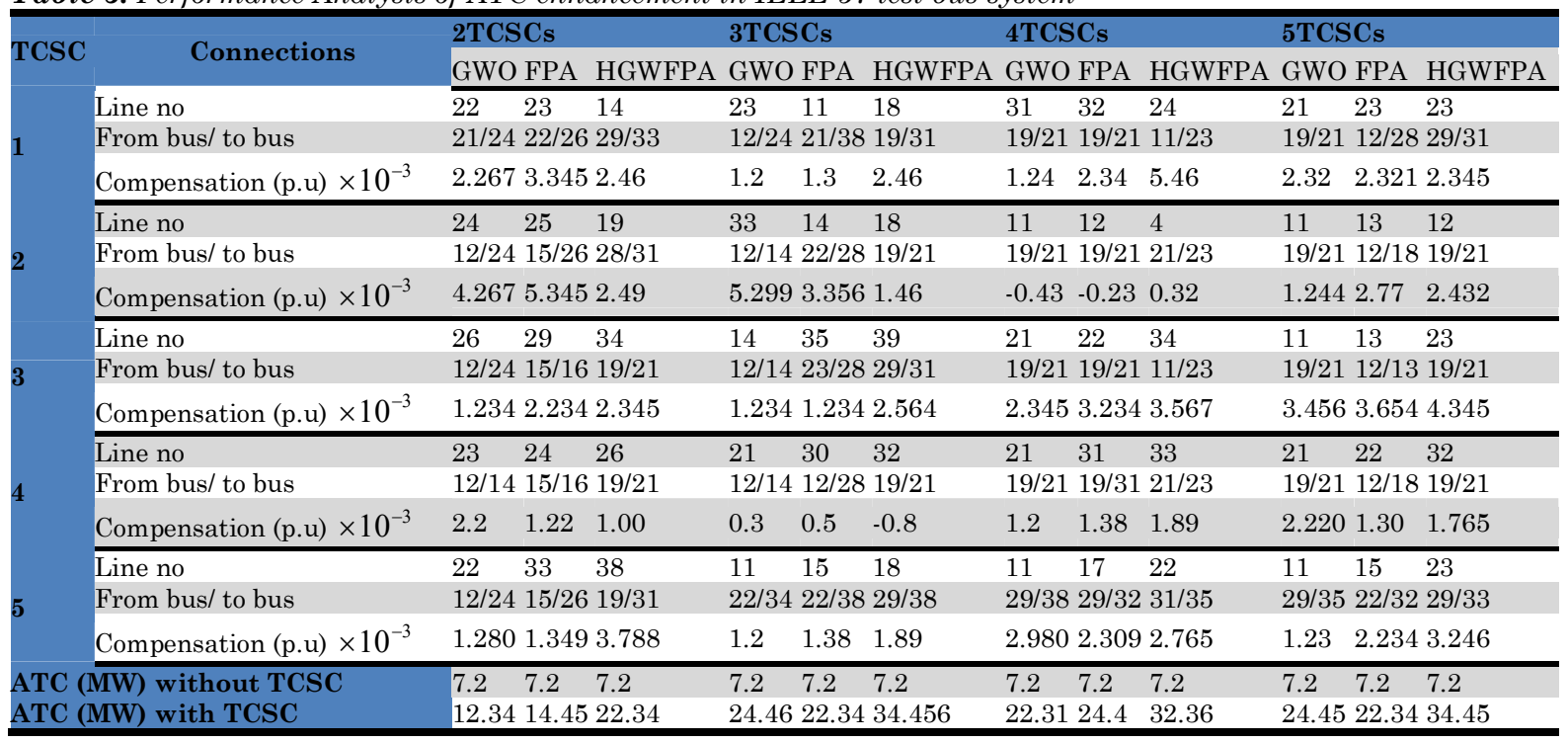

\subsection{Statistical Analysis}

Table 4 shows the performance analysis of IEEE 24, 30 and 57 bus systems. For the best-case scenario, the performance of proposed HGWFPA on IEEE 24 test bus system is $8.6 \%$ superior to GWO, 8.9\% superior to FPA. In IEEE 30 test bus system, the proposed method is $7.1 \%$ superior to GWO and $6.2 \%$ superior to FPA. The proposed HGWFPA in IEEE 57 test bus system is superior to conventional GWO and FPA. Likewise, the worst, mean, median, as well as standard deviation performance of ATC enhancement on IEEE 24, 30 and 57 test bus systems of proposed HGWFPA, is greater than existing GWO and FPA method.

Table 4. Statistical Analysis of Proposed and Conventional Methods

\begin{tabular}{|c|c|c|c|c|c|c|c|c|c|}
\hline \multirow{2}{*}{$\begin{array}{c}\text { Bus } \\
\text { Statistics }\end{array}$} & \multicolumn{3}{|c|}{ IEEE 24 bus system } & \multicolumn{3}{|c|}{ IEEE 30 bus system } & \multicolumn{3}{|c|}{ IEEE 57 bus system } \\
\hline & GWO & FPA & HGWFPA & GWO & FPA & HGWFPA & GWO & FPA & HGWFPA \\
\hline Best & 23 & 22 & 32 & 22 & 21 & 45 & 23 & 34 & 45 \\
\hline Worst & 82 & 83 & 82 & 75 & 74 & 74 & 23 & 22 & 23 \\
\hline Mean & 210.2 & 222.3 & 321.2 & 221.22 & 321.22 & 354.2 & 213.4 & 251.3 & 289.3 \\
\hline Median & 132.2 & 76.33 & 221.1 & 83.2 & 98.4 & 101.3 & 73.4 & 98.6 & 110.4 \\
\hline $\begin{array}{l}\text { Standard } \\
\text { Deviation }\end{array}$ & 112.3 & 22.1 & 221.3 & 44.32 & 22.4 & 54.6 & 23.4 & 33.5 & 57.8 \\
\hline
\end{tabular}

\section{Conclusion}

In general, ATC is improved to enhance the consistency of the power system. Nevertheless, ATC is minimized in few conditions while the power system is exaggerated by the practice of congested circuits as well as buses with inadequate voltage. Here, HGWFPA method was presented to optimize the maximum ATC in the power transmission system. Then, TCSC devices were exploited as a substitute of FACTS devices. Finally, the experimentation was performed on IEEE 24, 30 and 57 test bus systems. Furthermore, the statistical study of the proposed HGWFPA algorithm experimented on the three test bus system was compared with the existing GWO and FPA method. Ultimately, the outcome attained from the comparison was shown that the proposed HGWFPA method improves the measurement of ATC with maximum enhancement than the existing GWO and FPA method.

\section{Compliance with Ethical Standards}

Conflicts of interest: Authors declared that they have no conflict of interest.

Human participants: The conducted research follows the ethical standards and the authors ensured that they have not conducted any studies with human participants or animals. 


\section{References}

[1] RahulAgrawal,K.Bharadwaj, D.P.Kothari,"Population based evolutionary optimization techniques for optimal allocation and sizing of Thyristor Controlled Series Capacitor",Journal of Electrical Systems and Information Technology, vol. 5, no. 3, pp. 484-501, December 2018.

[2] Dillip Kumar Sahoo, Rabindra Kumar Sahu, G. T. Chandra Sekhar, Sidhartha Panda,"A novel modified differential evolution algorithm optimized fuzzy proportional integral derivative controller for load frequency control with thyristor controlled series compensator",Journal of Electrical Systems and Information Technology, vol. 5, no. 3,pp. 944-963, December 2018.

[3] M. B. Shafik, H. Chen, G. I. Rashed and R. A. El-Sehiemy, "Adaptive Multi Objective Parallel Seeker Optimization Algorithm for Incorporating TCSC Devices into Optimal Power Flow Framework," IEEE Access, vol. 7, pp. 36934-36947, 2019.

[4] O. Ziaee and F. F. Choobineh, "Optimal Location-Allocation of TCSC Devices on a Transmission Network," IEEE Transactions on Power Systems, vol. 32, no. 1, pp. 94-102, Jan. 2017.

[5] T. Nireekshana, G. Kesava Rao, S. Sivanaga Raju,Available transfer capability enhancement with FACTS using Cat Swarm Optimization,” Ain Shams Engineering Journal, vol 7, no. 1, pp. 159-167, March 2016.

[6] M. Venkateswara Rao, S. Sivanagaraju, Chintalapudi V. Suresh,"Available transfer capability evaluation and enhancement using various FACTS controllers: Special focus on system security",Ain Shams Engineering Journal, vol. 7, no. 1, pp. 191-207, March 2016.

[7] Ashwani Kumar, Jitendra Kumar,"ATC enhancement in electricity markets with GUPFC and IPFC - A comparison, International Journal of Electrical Power \& Energy Systems, vol. 81, pp. 469-482, October 2016.

[8] Zora Luburic, Hrvoje Pandzic,"FACTS devices and energy storage in unit commitment",International Journal of Electrical Power \& Energy Systems, vol. 104,pp. 311-325, January 2019.

[9] Samo Gasperic, Rafael Mihalic,"Estimation of the efficiency of FACTS devices for voltage-stability enhancement with PV area criteria”,Renewable and Sustainable Energy Reviews, vol. 105, pp. 144-156, , May 2019.

[10] R. Wibowo, N. Yorino, M. Eghbal, Y. Zoka, and Y. Sasaki, "Facts devices allocation with control coordination considering congestion relief and voltage stability," Power Systems, IEEE Transactions on, vol. 26, no. 4, pp. 2302-2310, Nov 2011.

[11] E. Ghahremani and I. Kamwa, "Optimal placement of multiple-type facts devices to maximize power system loadability using a generic graphical user interface," Power Systems, IEEE Transactions on, vol. 28, no. 2, pp. 764-778, May 2013.

[12] S. Xia, K. W. Chan, X. Bai, and Z. Guo, "Enhanced particle swarm optimisation applied for transient angle and voltage constrained discrete optimal power flow with flexible ac transmission system," Generation, Transmission Distribution, IET, vol. 9, no. 1, pp. 61-74, 2015.

[13] O. Ziaee and F. Choobineh, "Thyristor-controlled switch capacitor placement in large-scale power systems via mixed integer linear programming and taylor series expansion," in PES General Meeting I Conference Exposition, IEEE, pp. 1-5, July 2014.

[14] Rashidinejad M, Farahmand H, Fotuhi-Firuzabad M and Gharaveisi A A,"ATC enhancement using TCSC via artificial intelligent techniques", Electr. Power Syst. Res. 78(1): 11-20,2008.

[15] Khaburi M A and Haghifam M R,"A probabilistic modeling based approach for total transfer capability enhancement using FACTS devices". Int. J. Electr. Power. 32(1): 12-16,2010.

[16] Hiraki Y, Hiraiwa T and Iwamoto S," NAS battery system design and allocation for improvement of transient stability ATC",In: 10th International Power \& Energy Conference (IPEC), Chicago, pp. 190-195, 2012.

[17] Satoh T, Tanaka H and Iwamoto S," ATC Improvement by phase shifter application considering dynamic rating”, In: Power Symposium, NAPS “07 39th North American, Las Cruces, pp. 528-533, 2007.

[18] Jain T, Singh S N and Srivastava S C," Dynamic ATC enhancement through optimal placement of FACTS controllers," Electr. Power Syst. Res. vol.79 no(11): pp.1473-1482.

[19] De A, Mamanduru V K R, Gunasekaran A, Subramanian Nand Tiwari M K," Composite particle algorithm for sustainable integrated dynamic ship routing and scheduling optimization", Comput. Ind. Eng. 96: 201-215, 2016.

[20] Alexandridis A, Chondrodima E and Sarimveis H," Cooperative learning for radial basis function networks using particle swarm optimization. Appl. Soft Comput. 49: 485-497,2016.

[21] De A, Kumar S, Gunasekaran A and Tiwari M K, "Sustainable maritime inventory routing problem with time window constraints", Eng. Appl. Artif. Intell. 61: 77-95, 2017.

[22] Soleimani H and Kannan G," A hybrid particle swarm optimization and genetic algorithm for closed-loop supply chain network design in large-scale networks", Appl. Math. Model. 39(14): 3990-4012, 2015.

[23] De A, Awasthi A and Tiwari M K," Robust formulation for optimizing sustainable ship routing and scheduling problem”,IFAC-PapersOnLine. 48(3): 368-373, 2015.

[24] Li C-Y and Liu C-W," A new algorithm for available transfer capability computation”, Int J. Elec. Power. 24(2): 159-166, 2002.

[25] Vaithilingam C and Kumudini Devi R,"Available transfer capability estimation using Support Vector Machine",Int. J. Elec. Power. 47: (May): 387-393,2013.

[26] Sheng H and Chiang H-D,"CDFLOW: A practical tool for tracing stationary behaviors of general distribution networks,"IEEE Trans. Power Syst. 29(3): 1365-1371, 2014.

[27] Mirjalili, S., Mirjalili, S.M., Lewis, A.: Grey Wolf Optimizer. Adv. Eng. Softw. vol.69, pp.46-61, 2014.

[28] Yang, X.S."Flower pollination algorithm for global optimization". Lecture Notes in Computer Science including subseries Lecture Notes in Artificial Intelligence and Lecture Notes in Bioinformatics, pp. 240-249, 2012. 
[29] Ejebe G C, Tong J, Waight J G, Frame J G, Wang X and Tinney W F Available transfer capability calculations. IEEE Trans. Power Syst. vol. 13,no. 4, pp.1521-1527,199.

[30] Gurinderpal Singh, V.K. Jain \& Amanpreet Singh, "A prediction model for the smart Industries, aiding photovoltaic integrated greenhouse system in biogas heating", Computers \& Electrical Engineering, 2018.

[31] RT Satish, KT Reddy, "A technique to find optimal location for wavelet-based image watermarking using genetic algorithm", Machine Graphics \& Vision International Journal,vol. 20 (2), pp.173-196, 2011.

[32] SB Vinay Kumar, PV Rao, Manoj Kumar Singh,"Multi-culture diversity based self adaptive particle swarm optimization for optimal floorplanning",Multiagent and Grid Systems, vol14, no.1, pp.31-65, 2018.

[33] V Polepally, KS Chatrapati, "DEGSA-VMM: Dragonfly-based exponential gravitational search algorithm to VMM strategy for load balancing in cloud computing",Kybernetes, vol 47 (6), pp. 1138-1157, 2018. 